Three-dimensional measurements of non-diffusive fast ion transport in TORPEX

This content has been downloaded from IOPscience. Please scroll down to see the full text.

View the table of contents for this issue, or go to the journal homepage for more

Download details:

This content was downloaded by: riccipaolo

IP Address: 128.178.183.105

This content was downloaded on $30 / 11 / 2013$ at $14: 11$

Please note that terms and conditions apply. 


\title{
Three-dimensional measurements of non-diffusive fast ion transport in TORPEX
}

\author{
A Bovet, I Furno, A Fasoli, K Gustafson and P Ricci \\ École Polytechnique Fédérale de Lausanne (EPFL), Centre de Recherches en Physique des Plasmas \\ (CRPP), Association Euratom-Confédération Suisse, CH-1015 Lausanne, Switzerland \\ E-mail: alexandre.bovet@epfl.ch
}

Received 12 July 2013, in final form 11 September 2013

Published 28 November 2013

Online at stacks.iop.org/PPCF/55/124021

\begin{abstract}
In the basic plasma physics device TORPEX, progress in the fundamental understanding of supra-thermal ion transport is achieved by extensive sets of three-dimensional (3D) data, together with numerical simulations of supra-thermal ion tracers in fluid turbulent fields. A miniaturized lithium 6+ ion source injects fast ions with energies up to $1 \mathrm{KeV}$ and a double-gridded energy analyzer is used as a detector. The source is mounted on a toroidally movable system and the detector can be moved in the poloidal cross-section, allowing one to reconstruct 3D fast ion current profiles. Synchronous detection is used to enhance the signal-to-noise ratio. A modulated biasing voltage is applied to the fast ion source and an analog lock-in amplifier is used to demodulate the detector signal. The analog lock-in amplifier is specially designed to remove the capacitive noise associated with the voltage modulation. Radial transport of the fast ions, associated with plasma turbulence, is characterized. A synthetic diagnostic allows comparing the experimental results with numerical simulations of the fast ion transport in a global fluid simulation of the TORPEX plasma. A good agreement is shown.
\end{abstract}

(Some figures may appear in colour only in the online journal)

\section{Introduction}

In astrophysical plasmas, such as the solar wind or the magnetosphere, ions can be accelerated by wave-particle interactions to high energies $[1,2]$ and become harmful to spacecraft functioning. In fusion devices, supra-thermal ions may be created by fusion reactions or additional heating. Achieving a good confinement of these particles is required to reach burning conditions [3]. The understanding of fast ion transport is an essential issue for laboratory and astrophysical plasmas.

The transport of supra-thermal ions is generally nondiffusive [4-6]. Non-diffusive transport [7,8] is characterized by the time evolution of the mean-square displacement of the particles: $\sigma^{2}(t) \sim t^{\gamma}$, where $\sigma^{2}$ is the variance of the fast ion positions and $\gamma$ is the transport exponent. The transport regime is called superdiffusive or subdiffusive if $\gamma$ is greater or smaller than one, respectively. When $\gamma=1$, classical diffusion is recovered.
In this paper, we describe experimental and numerical investigations of fast ion transport conducted in the basic plasma device TORPEX [9] in a simple magnetized torus configuration [10,11]. TORPEX has a major radius of $1 \mathrm{~m}$ and a minor radius of $20 \mathrm{~cm}$. A small vertical magnetic field, $B_{v}=2 \mathrm{mT}$, superimposed on a toroidal magnetic field, $B_{t}=$ $74 \mathrm{mT}$, creates helicoidal field lines with $\nabla B$ and curvature terminating on the vessel. These are the first measurements of fast ion transport in a basic toroidal device. Similar measurements of fast ion transport have been performed previously in the linear device at the large plasma device (LAPD) [5,12]. The experimental setup, presented in section 2, consists of a fast ion source and a fast ion detector inserted in the TORPEX vacuum chamber. This setup permits a reconstruction of the time-average three-dimensional (3D) fast ion beam, revealing the main features of the fast ion dynamics, including the effect of the plasma turbulence. The turbulent transport of these fast ions is evaluated by measuring the radial width of the beam as a function of the toroidal distance traveled 
by the ions. The measurement analysis method is presented in section 3 .

Extensive numerical studies of the interaction between the electrostatic TORPEX turbulence and the fast ions are conducted by following tracer particles in the turbulent electric field provided by global fluid simulations. A synthetic diagnostic is used to benchmark the simulations, which are used to extract a value of the transport exponent. The theoretical framework and the numerical simulations are presented in section 4, while the synthetic diagnostic is presented in section 5 .

\section{Experimental setup}

\subsection{Fast ion source}

The source is based on a two grid accelerating system with a thermionic emitter similar to the one used at the LAPD at UCLA [13,14]. The emitter is made from lithium aluminosilicate, purchased from HeatWave Labs Inc [15]. The original design of the fast ion source $[16,17]$ has been improved to have a better electrical insulation of the different components by carving paths for the different electric wires directly in the boron nitride casing. To facilitate the assembly of the source, the original design of the boron nitride casing, consisting of a cylinder with a screw cap, has been modified to a design with two boron nitride pieces encasing the emitter and the grids, held by ceramic screws. Speeding up the mounting of the source is important since the ${ }^{6} \mathrm{Li}$ ions emitter has a relatively short life expectancy $(\sim 40 \mathrm{~h})$.

In order to perform 3D measurements of the fast ion beam, the source is mounted on a rail that allows it to be moved in the toroidal direction (figure 1). A schematic of the experimental setup is detailed in figure 1 of [14]. The source is moved between discharges and a high-resolution poloidal cross-section of the fast ion current profile is measured by the detector for each toroidal position of the source. The complete system is contained inside the vacuum chamber of TORPEX. The possibility of moving the source without having to open TORPEX and breaking the vacuum is essential in this experiment. Indeed, the lifetime of the emitter is greatly diminished when it is exposed to atmospheric pressure. An ultrasonic piezoelectric motor was chosen to move the source on the rail. Ultrasonic motors are particularly well suited to work in vacuum and magnetized environments since they do not need any greasing and do not embed permanent magnets.

\subsection{Fast ion detection}

The detector consists of two identical miniaturized gridded energy analyzers (GEA) allowing measurement of the fast ion energy and current $[14,16,17]$. The two GEAs face opposite directions so that differential detection is used to remove a significant part of the background noise. Measurements are made with two detectors positioned at different ports along the toroidal direction. Using two detectors allows doubling the number of measurements without having to open TORPEX.

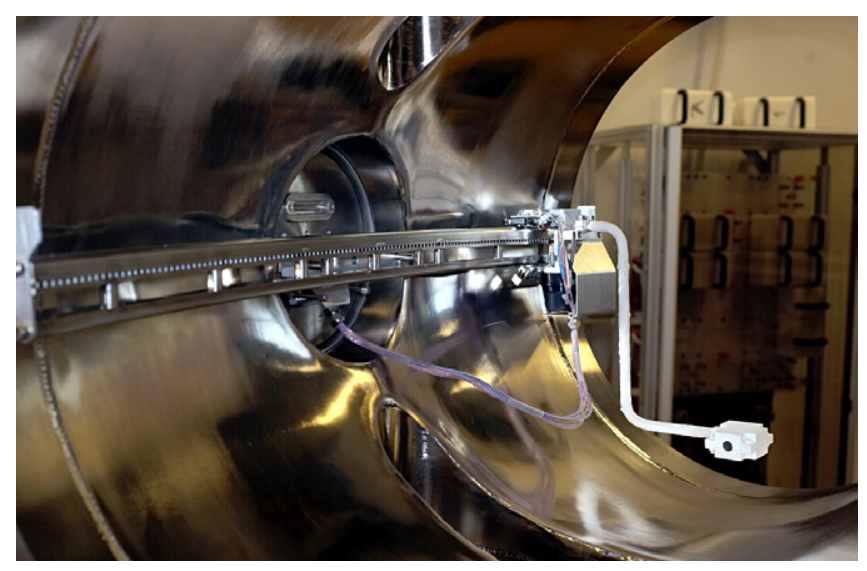

Figure 1. Photograph of the fast ion source mounted on the toroidally movable system in one of the TORPEX sectors. The source is positioned in the lower part of the device to maximize the distance traveled by the ions before they reach the upper wall because of their vertical drift.

As the fast ion current is extremely weak $(\sim 10 \mu \mathrm{A})$ and its signal is small compared to the background noise, a synchronous detection scheme is used to improve the signal-to-noise ratio [18]. In our case, the emitter bias is square-modulated by a reference signal (figure $2(b)$ ) at a given frequency $\left(f_{\text {ref }}=1073 \mathrm{~Hz}\right.$ ) chosen to avoid harmonics of the electrical grid $(50 \mathrm{~Hz})$. The fast ion current is therefore 'switched' on and off at this frequency, while the background noise remains independent of this modulation.

The detection is made using a phase-sensitive detector (PSD), also called a 'lock-in' amplifier, usually consisting of a switch operated at the reference frequency, an RC low-pass filter and a differential amplifier (figure 2(a)). The switch inverts the signal periodically, resulting in a signal consisting of the fast ion current and the background noise during the 'on' phase and only the inverted noise during the 'off' phase. The low-pass filter averages the resulting signal over many periods of the reference signal, effectively averaging-out the noisy part of the signal and giving a de signal proportional to the fast ion current. Unfortunately, in our setup, the modulation of the emitter results in undesired space-dependent capacitive effects in the detector (figure 2(c)). In order to remove this capacitive noise, a new lock in amplifier allowing one to set a dead-time has been developed. The dead-time is introduced with the help of a second switch (figure 2(a)). The two switches are opened and closed according to the pattern shown in figures $2(d)$ and (e). During the dead-time, the two switches are open and the common-mode rejection of the differential amplifier removes the capacitive effects from the output signal. The duration of the dead-time is adjusted to remove capacitive effects. The final signal is then integrated to obtain a dc output proportional to the fast ion current.

\section{Measurements analysis}

\subsection{Poloidal cross-section of the fast ion current}

Poloidal cross-sections of the fast ion current are reconstructed from the time-averaged current measurements. The detector 


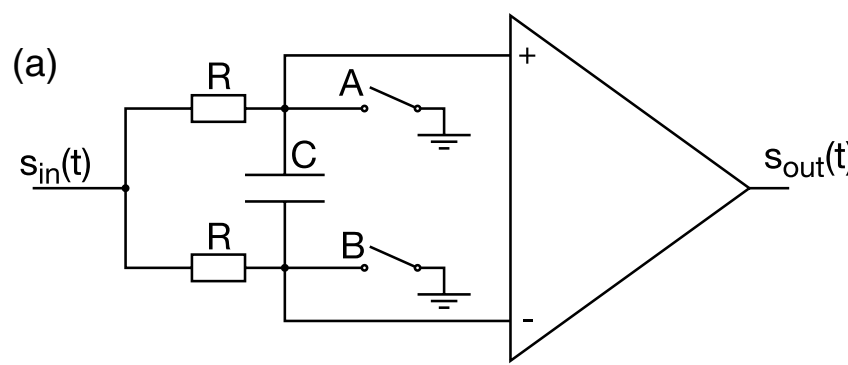

(b) Reference signal

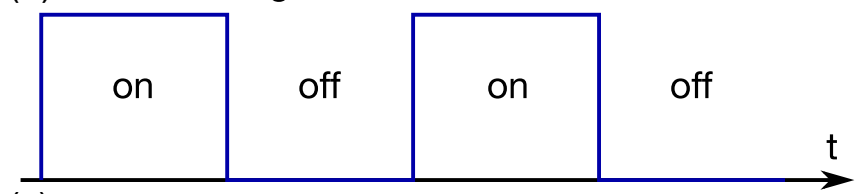

(c) $S_{\text {in }}(t)$

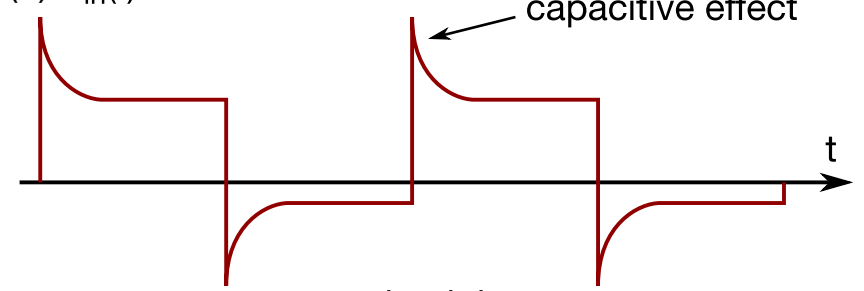

(d) Switch A

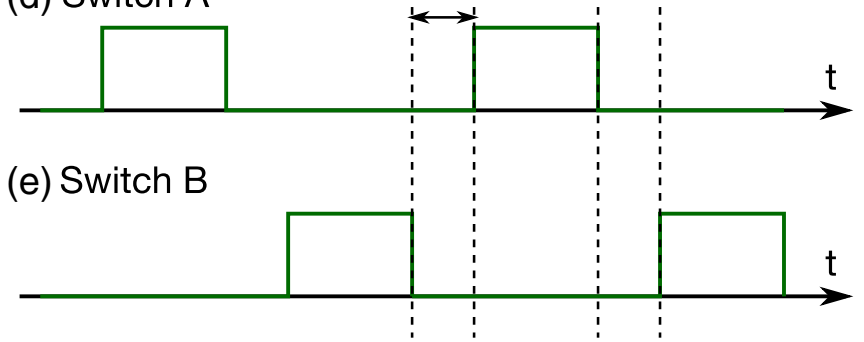

Figure 2. (a) Electronic diagram showing the principle of a lock-in amplifier with a dead-time. The system consists of two switches operated in a staggered pattern, an RC low-pass filter and a differential amplifier. $(b)$ Time trace of the reference signal used to modulate the source bias voltage. (c) The resulting signal in the detector with capacitive effects due to the voltage modulation of the source. $(d)$ and $(e)$ Switching pattern for the two switches. On these time traces, a signal value equal to zero represents an open switch.

is mounted on a motorized two-dimensional (2D) movable system [16]. A typical discharge lasts several minutes, during which the plasma is continuously sustained by injecting microwave power. The detector is moved in the poloidal plane allowing measurements at several positions. For example, the grey circles shown in figure 3 represent the measurement positions used to reconstruct the poloidal fast ion current profile (in color in the figure). The detector is moved in steps and remains at each position for at least $10 \mathrm{~s}$ to provide a statistically relevant time average. The time average is obtained using the analog lock-in amplifier presented in section 2.2. The poloidal current profile is then reconstructed by interpolating the scattered data points onto a $2 \mathrm{D}$ uniform grid with $1 \mathrm{~mm}$ spacing using the naturalneighbors method [19]. The offset arising from the analog lock-in amplifier is finally removed by computing its value as the average of all the interpolated data points that have a smaller value than the $5 \%$ of the maximum ion current value of the profile.

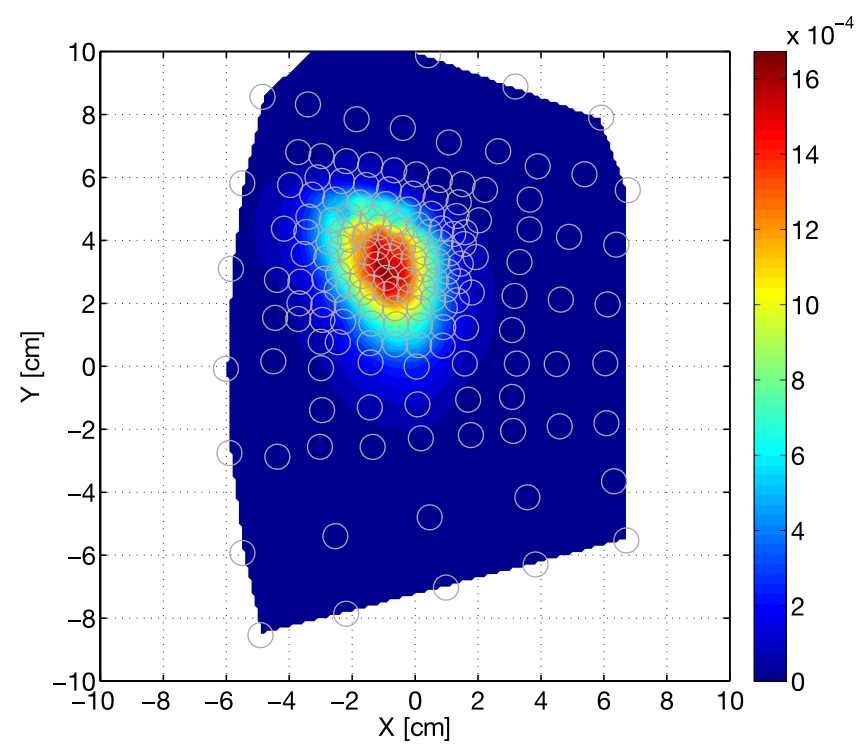

Figure 3. Poloidal fast ion current profile $[\mathrm{A}]$ at $2.2 \mathrm{~m}$ from the source for ions with an energy of $70 \mathrm{eV}$. The grey circles represent the measurements positions of the detector. The size of the circles represents the size of the detector inlet.

\subsection{Mean position and variance of the fast ion current poloidal profiles}

The motion of the fast ion beam is due to a combination of the gyromotion and the vertical drift caused by $\nabla B$ and curvature-drifts. In order to quantify the vertical drift, the mean vertical position of the fast ion current distribution is computed from the interpolated profiles (figure 3 ). The mean vertical position of the beam is computed by taking vertical slices of the profile every millimeter. In order to select the relevant portion of the profile, only slices that have a maximum value greater than the $30 \%$ of the global maximum value of the current are taken into account. Then, the mean vertical position is computed for each selected slice $j$ as

$$
\bar{y}_{j}=\frac{\sum_{i} y_{i} \cdot I_{i}}{\sum_{i} I_{i}},
$$

where the $y_{i}$ are the vertical positions along the $y$-axis and the $I_{i}$ are the corresponding ion current values. Finally, the mean vertical position of the beam profile, $\mu_{y}$, is computed as the average of all the $\bar{y}_{i}: \mu_{y}=\left\langle\bar{y}_{i}\right\rangle_{j}$. The error on the vertical mean position is given by the standard deviation of all the $\bar{y}_{i}$ and takes also the size of the detector into account: $e_{\mu_{y}}=\left[\frac{1}{N-1} \sum_{j=1}^{N}\left(\bar{y}_{j}-\mu_{y}\right)^{2}\right]^{1 / 2}+d / 2$, where $N$ is the number of slices and $d$ is the diameter of the detector inlet. This method is used for both experimental profiles and profiles from the synthetic diagnostic of numerical simulations (see section 5). A comparison of the mean vertical position as a function of the toroidal distance from the source, computed from experimental profiles and from profiles reconstructed from a synthetic diagnostic is shown in figure 4 . The vertical drift due to curvature and $\nabla B$-drifts is revealed.

In order to quantify the turbulent radial transport, the radial width, quantified by the standard deviation of the ion current distribution profile, is computed using a similar technique. 


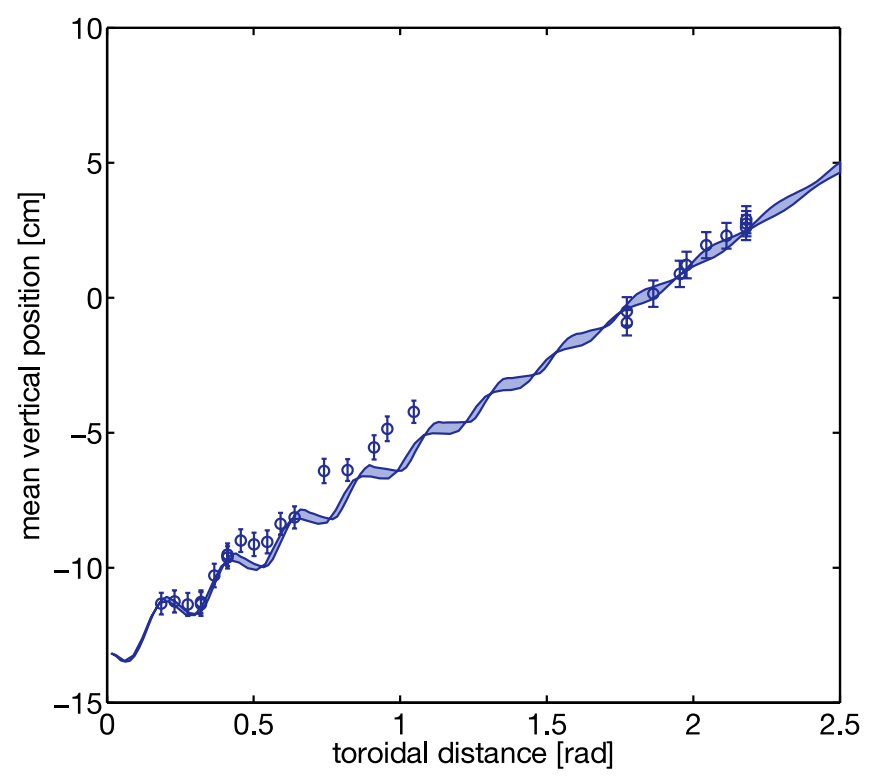

Figure 4. Mean vertical position as a function of the toroidal position for ions with an energy of $70 \mathrm{eV}$. The blue dots represent the experimental data and the band represents the value obtained from profiles made with the synthetic diagnostic, where initial injection parameters of the simulated ions trajectories are varied within reasonable values (see table 1 ) to obtain the shaded area. The vertical drift experienced by the fast ion beam is due to the curvature and the $\nabla B$ drifts. Along the toroidal axis, one radian corresponds to a distance of one meter.

Relevant horizontal slices are selected using the same criteria and the radial standard deviation of each slice is computed as

$$
\sigma_{R, j}=\left[\frac{\sum_{i}\left(x_{i}-\bar{x}_{j}\right)^{2} \cdot I_{i}}{\sum_{i} I_{i}}\right]^{1 / 2},
$$

where $x_{i}$ represents the radial positions and $\bar{x}_{j}$ represents the mean radial position of the slice $j$. The radial standard deviation of the entire profile is given as the average of the standard deviation of all the slices: $\sigma_{R}=\left\langle\sigma_{R, j}\right\rangle_{j}$. Its error is also given by the standard deviation of all the $\sigma_{R, j}$ added to the size of the detector: $e_{\sigma_{R}}=\left[\frac{1}{N-1} \sum_{j=1}^{N}\left(\sigma_{R, j}-\sigma_{R}\right)^{2}\right]^{1 / 2}+d / 2$. Figure 5 shows the radial variance of the experimental profiles as a function of the toroidal distance from the source and a comparison with the synthetic diagnostic. The oscillation of the beam width due to the gyromotion of the fast ions is visible, as is the spreading due to the plasma turbulence.

\section{Numerical investigations}

The TORPEX plasma has been subject to extensive modeling, including validation of a global plasma turbulence code $[20,21]$. These simulations have been performed in 2D and 3D limits of the drift-reduced Braginskii fluid equations, adapted to the TORPEX geometry and plasma parameters [22,23]. These simulations have confirmed that the fluctuation spectra in TORPEX plasmas are dominated by ideal interchange modes at sufficiently high values of the ratio of the vertical over toroidal magnetic field $B_{v} / B_{t}$. This is the case for all plasmas studied in this paper. For a theoretical understanding of the fast

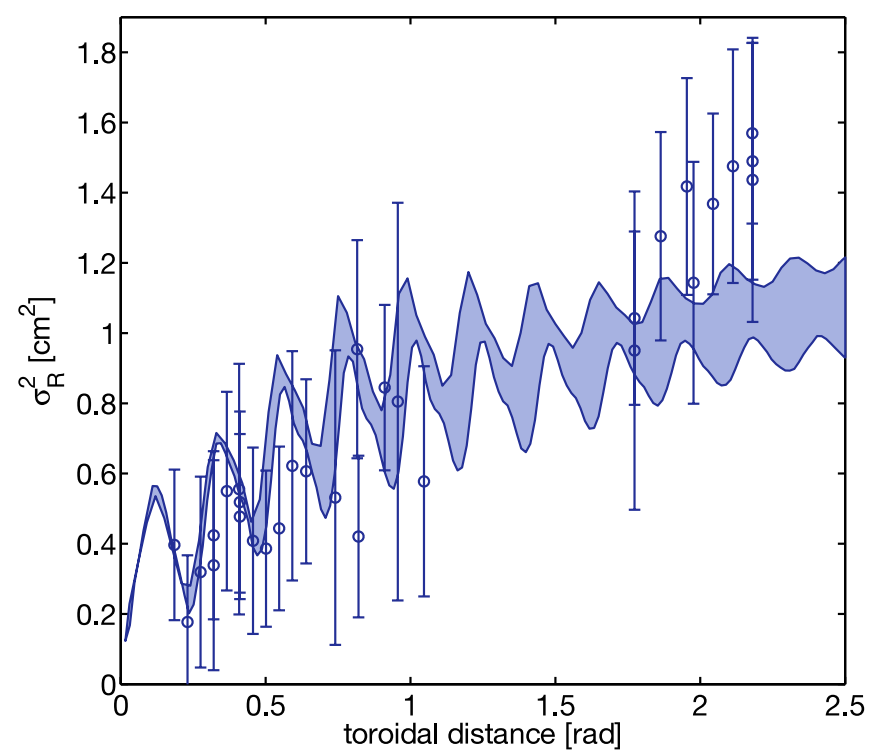

Figure 5. Radial variance of the fast ion current profiles as a function of toroidal distance for ions with an energy of $70 \mathrm{eV}$. The blue dots represent the experimental data and the line represents the value obtained from profiles obtained with the synthetic diagnostic, where initial injection parameters of the simulated ions' trajectories are varied within reasonable values (see table 1) to obtain the shaded area. The oscillations due to the gyromotion and the spreading due to the turbulence are clearly visible. Along the toroidal axis, one radian corresponds to a distance of one meter.

ion transport in this turbulent regime, a large number $\left(\sim 10^{5}\right)$ of tracer particles is injected in the simulated electrostatic field, with an injection geometry similar to that of the experiment; their trajectories are solved using the Lorentz force equation. These fast ion simulations explicitly include the charge and mass of the ${ }^{6} \mathrm{Li}$ ions, as well as the geometry of the helical magnetic field, which effectively causes curvature and $\nabla B$ drifts. The effect of gyroaveraging is also included with high accuracy.

A comprehensive theoretical study of the behavior of the fast ions as tracer particles $[6,24,25]$ has shown that the dispersion of fast ions in the radial direction is generally non-diffusive, with

$$
\sigma_{\delta R}^{2}(t) \sim t^{\gamma_{R}},
$$

where $\gamma_{R} \neq 1$. Here, $\sigma_{\delta R}^{2}(t)=\left\langle(\delta R-\langle\delta R\rangle)^{2}\right\rangle$ and $\delta R(t)=R(t)-R(0)$, where $R$ is the radial position of the ions. Initially, the dispersion of the fast ions is ballistic, with $\gamma_{R}=2$. This ballistic phase lasts approximately one gyroperiod, after which the interaction with the plasma becomes significant (see figure 6). A large number of different ensembles of fast ion simulations have revealed that the transport during the interaction phase is influenced by two mechanisms: gyroaveraging and drift-averaging [6]. The importance of those two effects was previously investigated as a function of two dimensionless quantities: the fast ion energy compared to the electron temperature, $E / T_{\mathrm{e}}$, and the amplitude of turbulent fluctuations relative to the electron temperature, $e \tilde{\phi} / T_{\mathrm{e}}$, where $\tilde{\phi}$ is the standard deviation of the plasma potential time series at the injection position. It was 


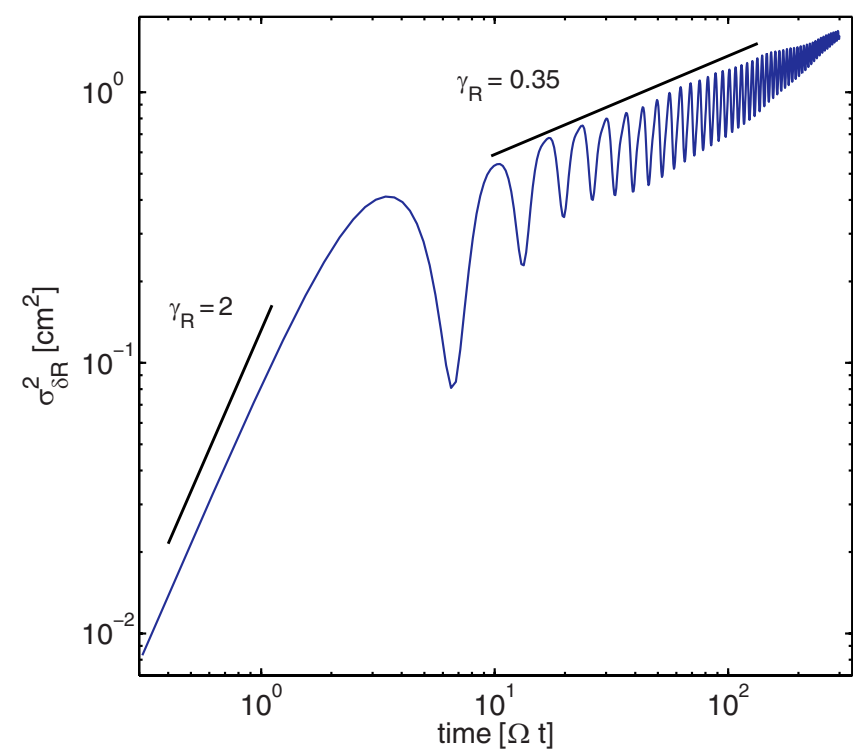

Figure 6. Variance of the radial positions of the simulated tracers as a function of time. The transport is initially ballistic $\left(\gamma_{R}=2\right)$ during approximately one gyroperiod, then it becomes subdiffusive, with $\left(\gamma_{R} \simeq 0.35\right)$, as the ions start to interact with the plasma.

seen that, depending on the value of these parameters, the value of $\gamma_{R}$ can go from superdiffusive $\left(\gamma_{R}>1\right)$ to subdiffusive $\left(\gamma_{R}<1\right)[6]$.

\section{Comparison between experiment and theory}

Comparisons between the fast ion current profiles measured in TORPEX and the simulations are made with the help of a synthetic diagnostic. This allows a characterization of experimentally measured fast ion beam profiles with the simulations. The synthetic diagnostic reconstructs time-averaged poloidal fast ion current profiles using the simulated tracer trajectories. To reconstruct the fast ion current at a fixed toroidal position, the poloidal plane is tiled in squares of a size comparable to the size of the fast ion detector inlet $(8 \mathrm{~mm} \times 8 \mathrm{~mm})$, reproducing the experimental resolution. To compare the time-average ion current measured from the continuously emitting source with the results of the simulations where the tracers are all launched simultaneously, the current is integrated in each tile by letting all the tracers cross the poloidal plane. The profile is then interpolated using the same method used for the experimental profiles. The mean vertical position and the radial variance are also computed in the same manner as for the experimental profiles (section 3).

Experiments are conducted with ions having an energy of $70 \mathrm{eV}$, injected in the blob region [26-28] of the TORPEX plasma. The ion velocity is mainly parallel to the magnetic field lines with a small perpendicular component. This is due to the beam divergence and to the small angle between the source orientation and the magnetic field lines. At the injection position, the electron temperature, measured by a triple probe [29], is $T_{\mathrm{e}} \simeq 1.3 \mathrm{eV}$, and the standard deviation of the plasma potential time series, indicating the level of fluctuations, is $\sigma_{\phi} \simeq 1 \mathrm{~V}$. The two detectors are used to measure
Table 1. Initial parameters of the simulated ion trajectories. The standard deviation of the Gaussian spreadings is also indicated. $\alpha$ is the angle between the source orientation and the horizontal plane and $\beta$ is the angle between the source orientation and the toroidal direction. Injection energy $E$, injection position $X$ and fluctuation amplitude, represented by the factor $\Xi$, were all varied within the indicated range to obtain the shaded area on figures 4 and 5 .

\begin{tabular}{lcclll}
\hline & $\alpha(\mathrm{rad})$ & $\beta(\mathrm{rad})$ & $E(\mathrm{eV})$ & $X(\mathrm{~cm})$ & $\Xi$ \\
\hline Inital value & -0.1 & -0.1 & {$[65,70]$} & {$[-2,-0.4]$} & {$[0.75,0.8]$} \\
Spreading & 0.08 & 0.08 & $5 \%$ & 0.012 & - \\
\hline
\end{tabular}

fast ion current profiles at a toroidal distance from the source, varying approximately from 0.2 to $2.2 \mathrm{~m}$.

Simulations of the 2D fluid code introduced in section 4 are performed with different values of the particle and heat sources to match the experimental plasma potential profile measured with the triple probe. The amplitude of the simulated plasma potential fluctuations is then multiplied by a factor $\Xi=0.75$ to match the one measured with the triple probe [9].

In order to compute the value of the radial transport exponent $\gamma_{R}$ from the experimental measurements, the synthetic diagnostic is used to verify the match between the experiment and the simulations. Figures 4 and 5 show the beam position and the beam variance computed from the experimental profiles and from the profiles reconstructed with the synthetic diagnostic for ions with an energy of $70 \mathrm{eV}$. A very good agreement is apparent. The agreement is quantified by the reduced chi-squared, computed as $\chi_{\text {red }}^{2}=\frac{1}{N} \sum_{i=1}^{N}\left[\left(y_{i, \exp }-\right.\right.$ $\left.\left.y_{i, \operatorname{sim}}\right) / e_{i}\right]^{2}$, where $N$ is the number of experimental points, the $y_{i, \exp }$ and $y_{i \text {,theo }}$ represent the experimental and theoretical values and the $e_{i}$ are the experimental errors. For the comparison of the vertical position of the beam, we find $\chi_{\text {red }}^{2}=2.4$, and for the comparison of the radial spreading, we find $\chi_{\text {red }}^{2}=0.9$. Initial injection parameters of the simulated ion trajectories are varied within the ranges indicated in table 1 , which allows one to quantify the simulation uncertainty, represented by the shaded area on figures 4 and 5 .

The transport exponent, $\gamma_{R}$, must be computed from the evolution of the radial position variance as a function of time (equation (3)). However, the experimental data are necessarily time-averaged and the radial spreading is experimentally accessible only as a function of toroidal distance. Since all ions have slightly different toroidal velocities, there is not a unique relation between toroidal position and time. Therefore, the transport exponent is computed from numerical simulations, where the dependence of the variance on time can be evaluated, that best match the experimental data in the sense detailed above. The plot of the radial tracer positions' variance as a function of time is shown in figure 6 , where one can see that for ions of $70 \mathrm{eV}$, the radial transport in the interaction phase is subdiffusive with a transport exponent $\gamma_{R} \simeq 0.35$. As previous numerical studies of fast ion transport in the same TORPEX regime, dominated by ideal interchange instability, have shown $[6,24]$, gyroaveraging is the principal mechanism responsible for subdiffusive transport in this case. When the size of the ion Larmor radius is comparable with the typical scale length of the turbulent eddies, ions effectively average out the turbulent electrostatic field over their gyromotion and the effect of the turbulence on their trajectories is reduced. 


\section{Conclusion}

Non-diffusive transport is revealed for ions with an energy of $70 \mathrm{eV}$ injected in the turbulent plasma of the TORPEX device. Measurements of the fast ion beam width as a function of the toroidal distance from the injection position are compared with the results of numerical simulations from a synthetic diagnostic and show a good agreement. The transport exponent is measured from the numerical simulations to be $\gamma_{R} \simeq 0.35$, corresponding to subdiffusive radial transport. Numerical investigations $[6,24]$ have identified gyroaveraging as the main mechanisms leading to subdiffusive transport. Further experiments will be conducted by varying the injection parameters, as, for example, the fast ion energy, in order to study the different regimes predicted by the cited numerical studies.

\section{Acknowledgments}

The authors would like to thank Fabio Avino and Joaquim Loizu for their helpful discussions and the CRPP technical team for their essential support. This work was supported in part by the Swiss National Science Foundation.

Euratom $@ 2013$

\section{References}

[1] Perri S and Zimbardo G 2009 Adv. Space Res. 44465

[2] Perrone D et al 2013 Space Sci. Rev. 178 233-70
[3] Fasoli A et al 2007 Nucl. Fusion 47 S264

[4] Greco A et al 2003 J. Geophys. Res. 1081395

[5] Zhou S et al 2010 Phys. Plasmas 17092103

[6] Gustafson K et al 2012 Phys. Rev. Lett. 108035006

[7] del Castillo-Negrete D et al 2004 Phys. Plasmas 113854

[8] Sanchez R et al 2008 AIP Conf. Proc. 211211

[9] Bovet A et al 2012 Nucl. Fusion 52094017

[10] Fasoli A et al 2010 Plasma Phys. Control. Fusion 52124020

[11] Fasoli A et al 2013 Nucl. Fusion 53063013

[12] Zhou S et al 2011 Phys. Plasmas 18082104

[13] Zhang Y et al 2007 Rev. Sci. Instrum. 78013302

[14] Heidbrink W W et al 2012 Plasma Phys. Control. Fusion 54124007

[15] Heatwave Labs Inc., alkali ion source documentation (www.cathode.com/pdf/tb-118.pdf)

[16] Plyushchev G et al 2006 Rev. Sci. Instrum. 77 10F503

[17] Plyushchev G 2009 Interaction of supra-thermal ions with turbulence in a magnetized toroidal plasma $P h D$ Thesis Lausanne

[18] Temple P A 1975 Am. J. Phys. 43801

[19] Sibson R 1981 A brief description of natural neighbour interpolation Interpreting Multivariate Data (Chichester: Wiley) pp 21-36

[20] Ricci P et al 2009 Phys. Plasmas 16055703

[21] Ricci P et al 2011 Phys. Plasmas 18032109

[22] Ricci P and Rogers B N 2009 Phys. Plasmas 16092307

[23] Ricci P and Rogers B N 2010 Phys. Rev. Lett. 104145001

[24] Gustafson K et al 2012 Phys. Plasmas 19062306

[25] Gustafson K and Ricci P 2012 Phys. Plasmas 19032304

[26] Podestà M et al 2008 Phys. Rev. Lett. 101045001

[27] Müller S H et al 2009 Plasma Phys. Control. Fusion 51055020

[28] Theiler C et al 2011 Phys. Plasmas 1855901

[29] Theiler C et al 2011 Rev. Sci. Instrum. 82013504 